\title{
Gene expression profiling and bioinformatics analysis of hereditary gingival fibromatosis
}

\author{
LEI FANG $^{1}$, YU WANG ${ }^{2}$ and XUEJUN CHEN ${ }^{1}$ \\ ${ }^{1}$ Department of Pathology and Pathophysiology, Jinzhou Medical University, Jinzhou, Liaoning 121001; \\ ${ }^{2}$ Department of Ultrasound in Medicine, Shanghai Jiaotong University Affiliated Sixth People's Hospital, \\ Shanghai Institute of Ultrasound in Medicine, Shanghai 200233, P.R. China
}

Received August 9, 2017; Accepted November 11, 2017

DOI: $10.3892 /$ br.2017.1031

\begin{abstract}
Hereditary gingival fibromatosis (HGF) is a benign, non-hemorrhagic and fibrous gingival overgrowth that may cover all or part of the teeth. It typically interferes with speech, lip closure and chewing, and can also be a psychological burden that affects the self-esteem of patients. Owing to high genetic heterogeneity, genetic testing to confirm diagnosis is not justified. It is therefore important to identify key signature genes and to understand the molecular mechanisms underlying HGF. The aim of the present study was to determine HGF-related genes and to analyze these genes through bioinformatics methods. A total of 249 differentially expressed genes (DEGs), consisting of 65 upregulated and 184 downregulated genes, were identified in the GSE4250 dataset of Gene Expression Omnibus (GEO) when comparing with the gums of HGF patients with those of healthy controls using the affy and limma packages in R. Subsequently, 28 enriched gene ontology terms were obtained from the Database for Annotation, Visualization and Integrated Discovery, and a protein-protein interaction (PPI) network was constructed and analyzed using STRING and Cytoscape. There were 99 nodes and 118 edges in the PPI network of these DEGs obtained through STRING. Among these nodes, 12 core genes were identified, of which the highest degree node was the gene for POTE ankyrin domain family member I. Collectively the results indicate that bioinformatics methods may provide effective strategies for predicting HGF-related genes and for understanding the molecular mechanisms of HGF.
\end{abstract}

\section{Introduction}

Gingival fibromatosis (GF) is a rare condition of gingival overgrowth, characterized by a slowly progressive, benign,

Correspondence to: Professor Xuejun Chen, Department of Pathology and Pathophysiology, Jinzhou Medical University, 40, Section 3, Songpo Road, Jinzhou, Liaoning 121000, P.R. China E-mail: thundempest@163.com

Key words: hereditary gingival fibromatosis, differentially expressed genes, protein-protein interaction, bioinformatics localized or generalized fibrous enlargement of maxillary and mandibular keratinized gingiva (1-3). GF may co-exist with various genetic syndromes, such as Rutherfurd syndrome, Cowden syndrome, Zimmerman-Laband syndrome, Murray-Puretic syndrome and hyaline fibromatosis syndrome, or occurs as an apparent isolated trait as non-syndromic hereditary GF (HGF) (1,4-6). HGF, also known as hereditary gingival hyperplasia or idiopathic gingival fibromatosis, is the most common genetic form of GF that is typically transmitted as an autosomal-dominant trait $(7,8)$. HGF affects males and females equally at an estimated incidence of 1 per 175,000 of the population $(1,9)$. As HGF is rare and benign, and due to an increase in the number of non-surgical treatments, it is difficult to collect large samples of HGF. To date, four loci, namely 2p22.1, 5q13-q22, 2p23.3-p22.3 and 11p15, have been associated with HGF, and a heterozygous frameshift mutation in Son of sevenless-1 (SOS1) has been reported as the cause of autosomal-dominant HGF in a family showing linkage to 2p21 (10). HGF exhibits an autosomal dominant inheritance pattern, although its penetrance and expressivity are variable (8). Indeed, $20 \%$ of cases have no family history of the condition (11). Diagnosis of HGF mainly depends on medical history, clinical examination, blood tests and histopathological evaluation of affected gingival tissue (1). However, owing to high genetic heterogeneity, genetic testing to confirm the diagnosis is not justified (12). It is therefore important to identify key signature genes and to understand the molecular mechanisms underlying HGF.

In the present study, microarray data from Gene Expression Omnibus (GEO, https://www.ncbi.nlm.nih.gov/geo/) (13) were used to identify differentially expressed genes (DEGs) in HGF. Subsequently, the identified DEGs were analyzed for enriched gene ontology (GO) terms and used for protein-protein interaction (PPI) network construction. Overall, this aimed to predict HGF-related genes and understand the molecular mechanisms of HGF.

\section{Materials and methods}

Gene expression microarray datasets. The gene expression microarray data of the GSE4250 dataset (14) were downloaded from the GEO database. There were 2 normal gingiva samples (normal gingiva replicatel and normal gingiva replicate2) and 
2 HGF patient gingiva simples (HGF patient gingiva replicate1 and HGF patient gingiva replicate2) in GSE4250. The platform of GSE4250 is GPL570: [HG-U133_Plus_2] Affymetrix Human Genome U133 Plus 2.0 Array.

Preprocessing of raw datasets. The raw gene expression data was preprocessed with R v3.4.0 (https://www.r-project. org/) (15). All gene expression values were obtained from the data of GSE4250 using the affy package (16). As some probes correspond to the same gene symbol, the average of the expression values of these probes was defined as the expression value of the gene. A total of 20,486 gene symbols were identified following preprocessing.

Screening of DEGs. DEGs of GSE4250 were identified using the limma package (17) in R. The screening conditions for DEGs were an adjusted $\mathrm{P}<0.05$ and $\operatorname{llog}$ fold change $(\mathrm{FC}) \mid>1$. The pheatmap package in $\mathrm{R}$ was used to generate a heatmap for the visualization of these DEGs.

Analysis of enriched GO terms and Kyoto Encyclopedia of Genes and Genomes (KEGG) pathways. Following DEG screening, the Database for Annotation, Visualization and Integrated Discovery (DAVID) v6.8 (https://david.ncifcrf. gov/) (18) was used to determine the enriched GO terms and Kyoto Encyclopedia of Genes and Genomes (KEGG) pathways of the DEGs. The enriched GO terms and KEGG pathways with $\mathrm{P}<0.05$ were selected.

Construction of PPI network. The STRING database (http:// string-db.org/) (19) was used for PPI network construction. The minimum required interaction score was set to 0.4 as default. Subsequently, Cytoscape software v3.5.0 (http://www. cytoscape.org/index.html) (20) was used for visualization of the PPI network, in which nodes represented genes and edges represented interactions between genes. The degree of a node was defined as the number of direct interactions between the corresponding gene and others in the network. Core genes were selected for based on a node degree $\geq 5$.

\section{Results}

DEGs. A total of 249 DEGs were identified from the gene expression microarray data of GSE4250 by comparing the HGF patient gingiva simples to the normal gingiva samples. There were 65 upregulated genes and 184 downregulated genes among these DEGs (Fig. 1). The top 10 up- and downregulated genes of the DEGs according to $\log \mathrm{FC}$ are listed in Table I.

Enriched GO terms of the DEGs. A total of 28 enriched GO terms were determined for the DEGs using the DAVID functional annotation tool. Table II lists the top 10 enriched GO terms of the DEGs according to the counts of genes; these terms were 'plasma membrane', 'extracellular exosome', 'extracellular region', 'extracellular space', 'structural molecule activity', 'apical plasma membrane', 'endosome', 'structural constituent of cytoskeleton', 'keratinocyte differentiation' and 'peptide cross-linking'.

KEGG pathway analysis was also attempted for the DEGs, but no significant pathways were identified.
Table I. Top 10 up- and downregulated differentially expressed genes in GSE4250.

\begin{tabular}{llcl}
\hline & Gene symbol & LogFC & P-value \\
\hline Upregulated & KRT2 & 3.943554 & $7.57 \mathrm{E}-04$ \\
& DSC1 & 3.942499 & $5.26 \mathrm{E}-07$ \\
& CLDN20 & 3.715159 & $6.95 \mathrm{E}-07$ \\
& ABCA12 & 3.460847 & $1.78 \mathrm{E}-05$ \\
& LCE2B & 3.324958 & $6.41 \mathrm{E}-07$ \\
& HTR3A & 3.267399 & $1.83 \mathrm{E}-06$ \\
& TGM7 & 2.900429 & $7.42 \mathrm{E}-04$ \\
& AADACL2 & 2.487881 & $3.00 \mathrm{E}-04$ \\
& BAMBI & 2.450982 & $1.18 \mathrm{E}-05$ \\
& ISL1 & 2.382094 & $8.71 \mathrm{E}-05$ \\
Downregulated & MYBPC1 & -4.429606 & $4.09 \mathrm{E}-02$ \\
& PLA2G2A & -4.354675 & $2.16 \mathrm{E}-02$ \\
& IGFL1 & -4.214503 & $4.66 \mathrm{E}-02$ \\
& CLCA4 & -4.092624 & $1.27 \mathrm{E}-02$ \\
& SERPINB4 & -3.554999 & $3.53 \mathrm{E}-02$ \\
& ANXA3 & -3.422065 & $4.39 \mathrm{E}-02$ \\
& GYS2 & -3.417237 & $3.18 \mathrm{E}-02$ \\
& CXCL6 & -3.390147 & $4.62 \mathrm{E}-02$ \\
& ECM1 & -2.728271 & $3.91 \mathrm{E}-02$ \\
& ADIRF & -2.386218 & $3.53 \mathrm{E}-02$
\end{tabular}

PPI network of the DEGs. There were 99 nodes and 118 edges in the PPI network of the DEGs generated using STRING (Fig. 2). Among these nodes, 12 core genes were identified, of which the highest degree node was the gene for POTE ankyrin domain family member I (POTEI). The other core genes were calbindin 2 (CALB2), fibroblast growth factor 13 and 8 (FGF13/8), loricrin (LOR), sphingosine-1-phosphate receptor 1 (S1PR1), Annexin A1 (ANXA1), complement C3 (C3), C-X-C motif chemokine ligand 6 (CXCL6), galanin receptor 3 (GALR3), kinase insert domain receptor (KDR) and regulator of G protein signaling 4 (RGS4; Table III).

\section{Discussion}

In the current periodontal diseases and conditions classification, which was developed by Armitage in 1999 (21), HGF is defined a benign, non-hemorrhagic and fibrous gingival overgrowth that may cover all or part of the teeth. HGF is also one of the subtypes of gingival lesions of genetic origin among gingival diseases $(6,22,23)$. HGF gingiva is typically pink in color and has a fibrous appearance and marked stippling without signs of inflammation, and covers the teeth partially or totally with a variable degree of severity, without affecting the bone $(6,23-26)$. It generally interferes with speech, lip closure and chewing, and may also become a psychological burden by affecting the self-esteem of patients (6). HGF presents an autosomal dominant inheritance pattern, although its penetrance and expressivity are variable. However, owing to high genetic heterogeneity, genetic testing to confirm the diagnosis is not justified. Thus, it is important to identify the key signature genes and to understand the molecular mechanisms 
Table II. Top 10 enriched GO terms of differentially expressed genes in GSE4250.

\begin{tabular}{|c|c|c|c|c|}
\hline Category & Term & Description & Count & P-value \\
\hline $\mathrm{CC}$ & GO:0005886 & Plasma membrane & 58 & $2.48 \mathrm{E}-02$ \\
\hline $\mathrm{CC}$ & GO:0070062 & Extracellular exosome & 56 & $5.82 \mathrm{E}-06$ \\
\hline $\mathrm{CC}$ & GO:0005576 & Extracellular region & 32 & $1.24 \mathrm{E}-03$ \\
\hline $\mathrm{CC}$ & GO:0005615 & Extracellular space & 25 & $1.14 \mathrm{E}-02$ \\
\hline MF & GO:0005198 & Structural molecule activity & 13 & $1.56 \mathrm{E}-05$ \\
\hline $\mathrm{CC}$ & GO:0016324 & Apical plasma membrane & 10 & 4.64E-03 \\
\hline $\mathrm{CC}$ & GO:0005768 & Endosome & 8 & $1.15 \mathrm{E}-02$ \\
\hline $\mathrm{MF}$ & GO:0005200 & Structural constituent of cytoskeleton & 7 & $1.23 \mathrm{E}-03$ \\
\hline $\mathrm{BP}$ & GO:0030216 & Keratinocyte differentiation & 7 & $1.90 \mathrm{E}-04$ \\
\hline $\mathrm{BP}$ & GO:0018149 & Peptide cross-linking & 7 & $1.75 \mathrm{E}-05$ \\
\hline
\end{tabular}

GO, gene ontology; CC, cellular component; MF, molecular function; BP, biological process.

of HGF. As an important discipline of biological science, bioinformatics analysis employs scientific resources for research purposes (27), and is considered an efficient method for predicting disease-related genes.

In the present study, 249 DEGs were identified, consisting of 65 upregulated and 184 downregulated genes, in the GSE4250 dataset. Notably, among the top 10 upregulated genes of the DEGs, the gene encoding bone morphogenetic protein and activin membrane bound inhibitor has previously been associated with fibromatosis (28). The DEGs were subsequently assessed by GO enrichment and PPI network analyses. The top 10 enriched GO terms of the DEGs were 'plasma membrane', 'extracellular exosome', 'extracellular region', 'extracellular space', 'structural molecule activity', 'apical plasma membrane', 'endosome', 'structural constituent of cytoskeleton', 'keratinocyte differentiation' and 'peptide cross-linking'. These results indicated that HGF-related enriched GO terms are principally associated with cell growth and tissue hyperplasia. Histologically, HGF is characterized by the growth and hyperplasia of gingival epithelial cells (22). Straka et al (29) observed in HGF that some collagen fibrils exhibited loops in the gingival lamina propria and identified the presence of empty perinuclear space in the cytoplasm of epithelial cells. These findings may relate to the enriched GO terms. The GO enrichment may indicate targets for research and guide studies on HGF-related genes based on the enriched GO terms. KEGG pathways were also evaluated based on the DEGs, but no significant pathways were identified. The method of DEG screening or the algorithms of the tools used may have lead to this result. From the PPI network of the DEGs, 12 core genes were identified that may serve critical roles in HGF. Among these core genes, POTEI had the highest node degree. However, to the best of our knowledge, there are no reports on the potential role of POTEI in HGF, though this may be due to the general lack of studies on HGF-related genes. Furthermore, while it has not been reported that CALB2, is directly associated with HGF, Barak et al (30) documented that calretinin encoded by the CALB2 gene may be an important immunohistochemical marker in other benign and malignant fibromatosis. The FGF family also serve an important role in fibroblast growth (31). Meanwhile, Lee et al (32) reported that
Table III. Differentially expressed genes in the protein-protein interaction network and their corresponding degree.

\begin{tabular}{lrlllr}
\hline Gene & Degree & Gene & Degree & Gene & Degree \\
\hline POTEI & 15 & CHP2 & 2 & FLRT3 & 1 \\
CALB2 & 7 & DHRS9 & 2 & GDA & 1 \\
FGF13 & 7 & GUCY2 & 2 & GRPEL2 & 1 \\
FGF8 & 7 & HS3ST1 & 2 & GYS2 & 1 \\
LOR & 7 & HS6ST2 & 2 & IGFL1 & 1 \\
S1PR1 & 7 & HTR3A & 2 & IGSF10 & 1 \\
ANXA1 & 6 & LRFN3 & 2 & KRT2 & 1 \\
C3 & 6 & NGFR & 2 & KRTAP3-2 & 1 \\
CXCL6 & 5 & OMA1 & 2 & LCE2B & 1 \\
GALR3 & 5 & OXT & 2 & MEDAG & 1 \\
KDR & 5 & PAX9 & 2 & MYBPC1 & 1 \\
RGS4 & 5 & PCSK1 & 2 & NEXN & 1 \\
DLX5 & 4 & PLA2G2A & 2 & NPR3 & 1 \\
GAPDHS & 4 & SLC34A2 & 2 & NR1D2 & 1 \\
ISL1 & 4 & SLC5A1 & 2 & OR10H1 & 1 \\
LPIN1 & 4 & SLC9A3R2 & 2 & OR1D2 & 1 \\
MSX2 & 4 & SPINK6 & 2 & PGM5 & 1 \\
RASL12 & 4 & SRGAP2 & 2 & PKP2 & 1 \\
RHOD & 4 & TM4SF1 & 2 & PLN & 1 \\
BBS10 & 3 & TPM1 & 2 & PNRC1 & 1 \\
DSC1 & 3 & TRIP10 & 2 & RGMA & 1 \\
GNL3 & 3 & TSPAN6 & 2 & RHCG & 1 \\
GPC6 & 3 & ARNTL & 1 & RPL18A & 1 \\
NKX2-3 & 3 & ATP6V0A4 & 1 & RSPO3 & 1 \\
POLR3F & 3 & BAMBI & 1 & RSPO4 & 1 \\
PPL & 3 & CEL & 1 & SCEL & 1 \\
SCD & 3 & CLDN20 & 1 & SDPR & 1 \\
TGM1 & 3 & CLDN5 & 1 & SMTNL2 & 1 \\
TUBB6 & 3 & CYP2J2 & 1 & SOD2 & 1 \\
ABCA12 & 2 & DUSP5 & 1 & SPHK1 & 1 \\
AOX1 & 2 & ECM1 & 1 & SPINK13 & 1 \\
AWAT1 & 2 & ELOVL4 & 1 & TEPP & 1 \\
CD59 & 2 & EPPK1 & 1 & ZBTB16 & 1 \\
\hline & & & & & \\
\hline
\end{tabular}

Core genes were selected for based on a node degree $\geq 5$. 


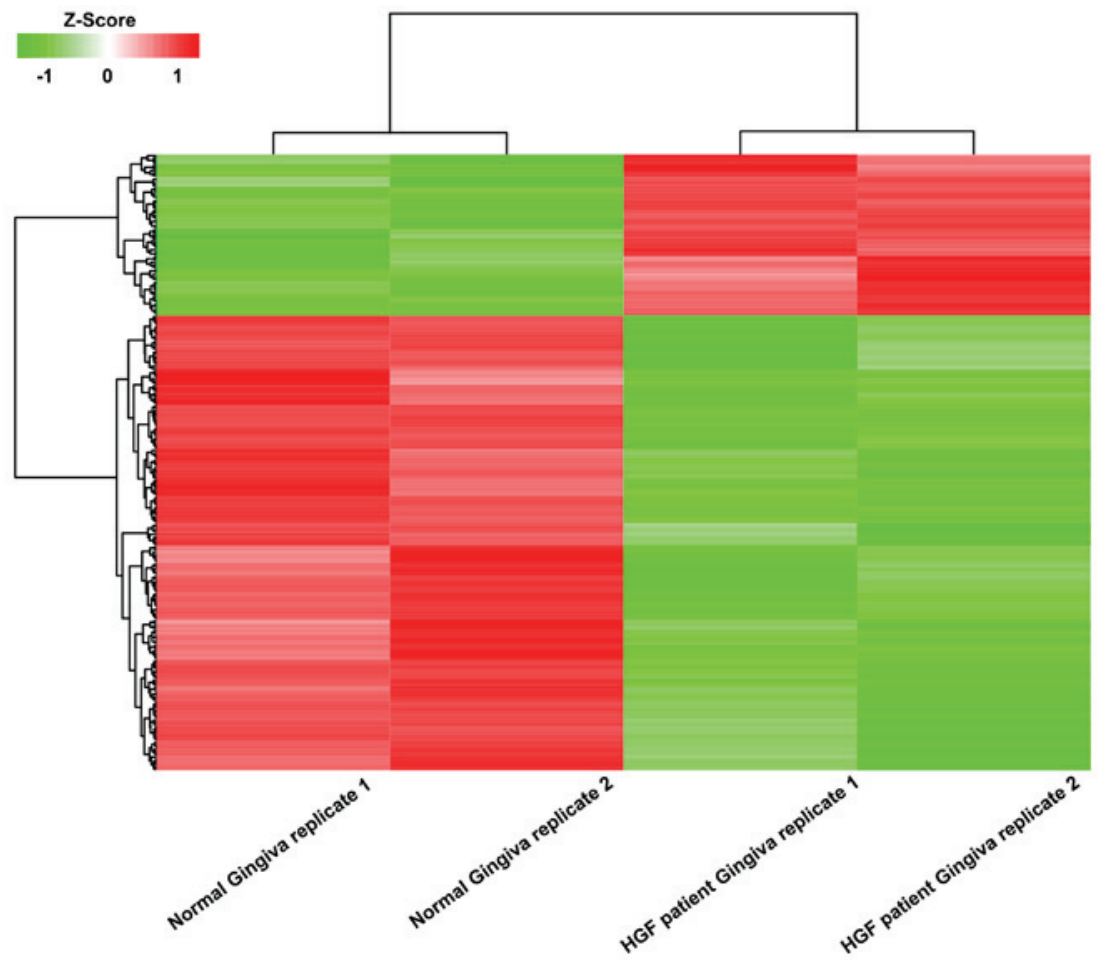

Figure 1. Heatmap of differentially expressed genes in GSE4250. Rows represent genes and columns represent samples. The heatmap is color-coded based on Z-score; red represents high expression value and green represents low expression value.

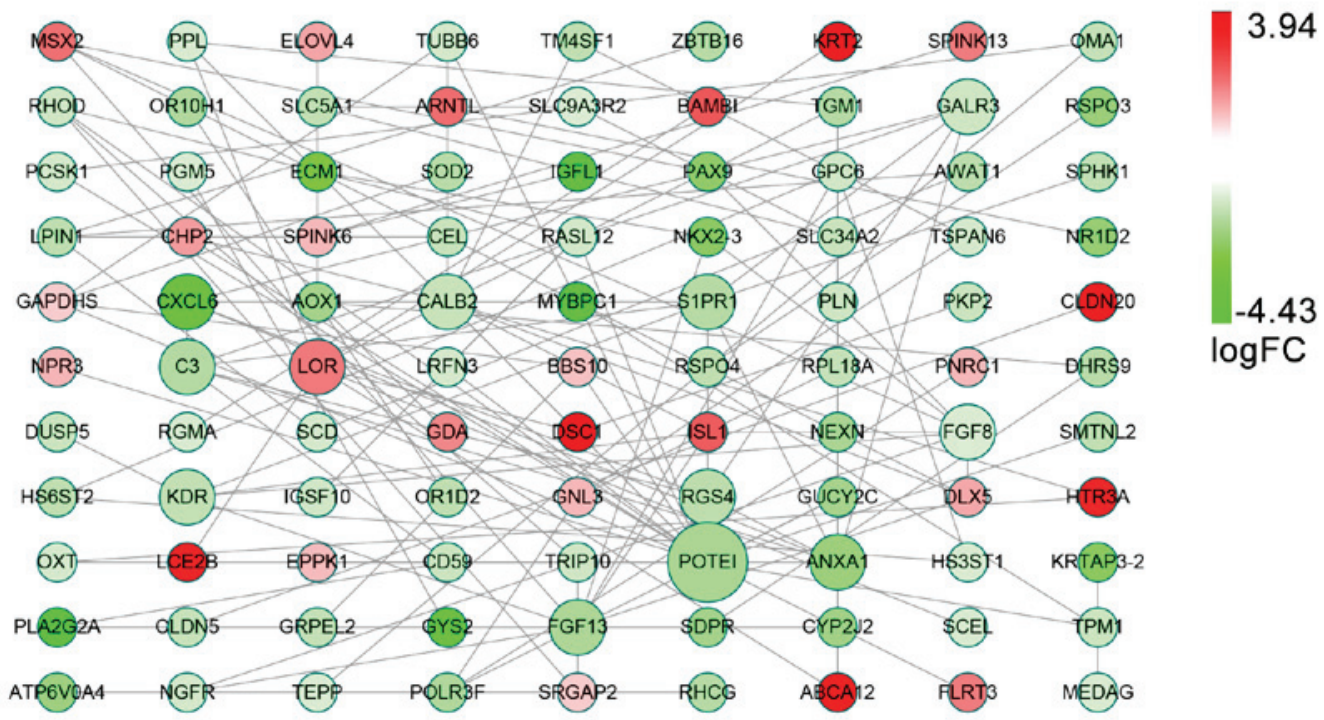

Figure 2. Protein-protein interaction network of differentially expressed genes in GSE4250. The color of each node represents the logFC value of the corresponding gene; the size of each node represents the degrees (connections) of the corresponding gene with others in the network. FC, fold-change.

LOR was important in keratinocyte differentiation in a study on the cell envelope of normal human oral keratinocytes. C3 has also been associated with fibrous papule development (33). Although reports on the functions of these core genes are limited, they may serve important roles in HGF.

In the present study, genes reported previously, including SOS1 (10), calcium/calmodulin-dependent protein kinase IV and adenosine triphosphate-binding cassette subfamily A member 5 (1), were not identified. This may have been due to the tools employed and the restricted screening parameters, as well as the limited scope of research on HGF. A crucial limitation of the present study was the small number of samples with and without HGF. Therefore, larger datasets and further experiments, for instance using reverse transcription-quantitative polymerase chain reaction, are required to validate the present results. Bioinformatics analysis is an efficient method for predicting potential diagnostic and therapeutic targets. However, the increased number of data mining and analytical tools and algorithms poses a challenge (34), as results for the same data using different bioinformatics tools may vary. 
Furthermore, the predictions require verification through experimental and clinical methods.

In summary, the prediction of potential diagnostic and therapeutic targets in diseases using bioinformatics methods is an efficient strategy in clinical research, though also poses a number of challenges. In the present study, a public dataset of GEO was used to analyze the potential diagnostic and therapeutic targets of HGF. The current predictions of potential diagnostic and therapeutic targets now require verification through experimental methods in cell and animal models prior to clinical trials.

\section{References}

1. Gawron K, Łazarz-Bartyzel K, Potempa J and Chomyszyn-Gajewska M: Gingival fibromatosis: Clinical, molecular and therapeutic issues. Orphanet J Rare Dis 11: 9, 2016.

2. Jayachandran M, Kapoor S and Mahesh R: Idiopathic gingival fibromatosis rehabilitation: A case report with two-year followup. Case Rep Dent 2013: 513153, 2013.

3. Mohan RP, Verma S, Agarwal N and Singh U: Non-syndromic hereditary gingival fibromatosis. BMJ Case Rep 2013 pii: bcr2012008542, 2013.

4. Tripathi AK, Dete G, Saimbi CS and Kumar V: Management of hereditary gingival fibromatosis: A 2 years follow-up case report. J Indian Soc Periodontol 19: 342-344, 2015.

5. Zhou M, Xu L and Meng HX: Diagnosis and treatment of a hereditary gingival fibromatosis case. Chin J Dent Res 14: 155-158, 2011.

6. Häkkinen L and Csiszar A: Hereditary gingival fibromatosis: Characteristics and novel putative pathogenic mechanisms. J Dent Res 86: 25-34, 2007.

7. Takagi M, Yamamoto H, Mega H, Hsieh KJ, Shioda S and Enomoto S: Heterogeneity in the gingival fibromatoses. Cancer 68: 2202-2212, 1991.

8. Majumder P, Nair V, Mukherjee M, Ghosh S and Dey SK: The autosomal recessive inheritance of hereditary gingival fibromatosis. Case Rep Dent 2013: 432864, 2013.

9. Odessey EA, Cohn AB, Casper F and Schechter LS: Hereditary gingival fibromatosis: Aggressive 2-stage surgical resection in lieu of traditional therapy. Ann Plast Surg 57: 557-560, 2006.

10. Hart TC, Zhang Y, Gorry MC, Hart PS, Cooper M, Marazita ML, Marks JM, Cortelli JR and Pallos D: A mutation in the SOS1 gene causes hereditary gingival fibromatosis type 1 . Am J Hum Genet 70: 943-954, 2002.

11. Harrison M, Odell EW, Agrawal M, Saravanamuttu R and Longhurst P: Gingival fibromatosis with prune-belly syndrome. Oral Surg Oral Med Oral Pathol Oral Radiol Endod 86: 304-307, 1998.

12. Ye X, Shi L, Yin W, Meng L, Wang QK and Bian Z: Further evidence of genetic heterogeneity segregating with hereditary gingival fibromatosis. J Clin Periodontol 36: 627-633, 2009.

13. Clough E and Barrett T: The Gene Expression Omnibus Database. Methods Mol Biol 1418: 93-110, 2016

14. Zhu Y, Zhang W, Huo Z, Zhang Y, Xia Y, Li B, Kong X and Hu L: A novel locus for maternally inherited human gingival fibromatosis at chromosome 11p15. Hum Genet 121: 113-123, 2007.

15. Team RDC: R: A Language and Environment for Statistical. Computing 14: 12-21, 2011.
16. Gautier L, Cope L, Bolstad BM and Irizarry RA: affy--analysis of Affymetrix GeneChip data at the probe level. Bioinformatics 20: 307-315, 2004.

17. Ritchie ME, Phipson B, Wu D, Hu Y, Law CW, Shi W and Smyth GK: limma powers differential expression analyses for RNA-sequencing and microarray studies. Nucleic Acids Res 43: e47, 2015 .

18. Huang W, Sherman BT and Lempicki RA: Systematic and integrative analysis of large gene lists using DAVID bioinformatics resources. Nat Protoc 4: 44-57, 2009.

19. Szklarczyk D, Franceschini A, Wyder S, Forslund K, Heller D, Huerta-Cepas J, Simonovic M, Roth A, Santos A, Tsafou KP, et al: STRING v10: Protein-protein interaction networks, integrated over the tree of life. Nucleic Acids Res 43 (D1): D447-D452, 2015.

20. Su G, Morris JH, Demchak B and Bader GD: Biological network exploration with Cytoscape 3. Curr Protoc Bioinformatics 47: $1-24,2014$

21. Armitage GC: Development of a classification system for periodontal diseases and conditions. Ann Periodontol 4: 1-6, 1999.

22. Coletta RD and Graner E: Hereditary gingival fibromatosis: A systematic review. J Periodontol 77: 753-764, 2006.

23. Tipton DA, Howell KJ and Dabbous MK: Increased proliferation, collagen, and fibronectin production by hereditary gingival fibromatosis fibroblasts. J Periodontol 68: 524-530, 1997.

24. Ramer M, Marrone J, Stahl B and Burakoff R: Hereditary gingival fibromatosis: identification, treatment, control. Journal of the American Dental Association 127: 493-495, 1996.

25. Laband PF, Habib G and Humphreys GS: Hereditary gingival fibromatosis. report of an affected family with associated splenomegaly and skeletal and soft-tissue abnormalities. Oral Surg Oral Med Oral Pathol 17: 339-351, 1964.

26. Araujo CS, Graner E, Almeida OP, Sauk JJ and Coletta RD: Histomorphometric characteristics and expression of epidermal growth factor and its receptor by epithelial cells of normal gingiva and hereditary gingival fibromatosis. J Periodontal Res 38: 237-241, 2003.

27. Karikari TK: Bioinformatics in Africa: The Rise of Ghana? PLOS Comput Biol 11: e1004308, 2015.

28. Kitazawa S, Kitazawa R, Obayashi C and Yamamoto T: Desmoid tumor with ossification in chest wall: possible involvement of BAMBI promoter hypermethylation in metaplastic bone formation. J Bone Miner Res 20: 1472-1477, 2005.

29. Straka M, Danisovic L, Bzduch V, Polak S and Varga I: The significance of electron microscopic examination of gingiva in cases of Hunter syndrome and hereditary gingival fibromatosis. Neuro Endocrinol Lett 37: 353-360, 2016.

30. Barak S, Wang Z and Miettinen M: Immunoreactivity for calretinin and keratins in desmoid fibromatosis and other myofibroblastic tumors: A diagnostic pitfall. Am J Surg Pathol 36: 1404-1409, 2012.

31. Ornitz DM and Itoh N: The Fibroblast Growth Factor signaling pathway. Wiley Interdiscip Rev Dev Biol 4: 215-266, 2015.

32. Lee CH, Marekov LN, Kim S, Brahim JS, Park MH and Steinert PM: Small proline-rich protein 1 is the major component of the cell envelope of normal human oral keratinocytes. FEBS Lett 477: 268-272, 2000.

33. Torre K, Larsen A, Kristjansson A and Murphy M: Basal cell carcinoma, arising within a granular cell-type fibrous papule. J Cutan Pathol 43: 1245-1247, 2016.

34. Charoentong P, Angelova M, Efremova M, Gallasch R, Hack1 H, Galon J and Trajanoski Z: Bioinformatics for cancer immunology and immunotherapy. Cancer Immunol Immunother 61: 1885-1903, 2012. 